\title{
Quantile stable mechanisms
}

Chen, Peter ; Egesdal, Michael ; Pycia, Marek ; Yenmez, M Bumin ; Pycia, Marek G

\begin{abstract}
We introduce a new class of matching mechanisms-quantile stable mechanisms-that generate stable matchings that can be seen as a compromise between sides of a two-sided market. We show that responsiveness is a sufficient condition for the existence of such mechanisms and that all such mechanisms are distinct. We also analyze the manipulability of these mechanisms by market participants.
\end{abstract}

DOI: https://doi.org/10.3390/g12020043

Posted at the Zurich Open Repository and Archive, University of Zurich

ZORA URL: https://doi.org/10.5167/uzh-205682

Journal Article

Published Version

(c) (1)

The following work is licensed under a Creative Commons: Attribution 4.0 International (CC BY 4.0) License.

Originally published at:

Chen, Peter; Egesdal, Michael; Pycia, Marek; Yenmez, M Bumin; Pycia, Marek G (2021). Quantile stable mechanisms. Games, 12(2):43.

DOI: https://doi.org/10.3390/g12020043 


\title{
Article
}

\section{Quantile Stable Mechanisms}

\author{
Peter Chen ${ }^{1, \dagger}{ }^{\dagger}$, Michael Egesdal ${ }^{2}$, Marek Pycia ${ }^{3, *}$ and M. Bumin Yenmez ${ }^{4}$ \\ 1 Analysis Group, Chicago, IL 60601, USA; peter.chen@analysisgroup.com \\ 2 Airbnb, Oakland, CA 94611, USA; mike.egesdal@airbnb.com \\ 3 Department of Economics, University of Zurich, 8006 Zurich, Switzerland \\ 4 Department of Economics, Boston College, Chestnut Hill, MA 02467, USA; bumin.yenmez@bc.edu \\ * Correspondence: marek.pycia@econ.uzh.edu \\ + The authors contributed equally to this work.
}

check for

updates

Citation: Chen, P.; Egesdal, M.; Pycia,

M.; Yenmez, M.B. Quantile Stable

Mechanisms. Games 2021, 12, 43.

https://doi.org/10.3390/g12020043

Academic Editor: Antonio Nicolò

Received: 15 April 2021

Accepted: 27 April 2021

Published: 17 May 2021

Publisher's Note: MDPI stays neutral with regard to jurisdictional claims in published maps and institutional affiliations.

Copyright: (c) 2021 by the authors. Licensee MDPI, Basel, Switzerland. This article is an open access article distributed under the terms and conditions of the Creative Commons Attribution (CC BY) license (https:// creativecommons.org/licenses/by/ $4.0 /)$.

\begin{abstract}
We introduce a new class of matching mechanisms—quantile stable mechanisms—that generate stable matchings that can be seen as a compromise between sides of a two-sided market. We show that responsiveness is a sufficient condition for the existence of such mechanisms and that all such mechanisms are distinct. We also analyze the manipulability of these mechanisms by market participants.
\end{abstract}

Keywords: matching; contracts; auctions; mechanisms; incentives

\section{Introduction}

We study a two-sided matching market between firms and workers. Each agent can simultaneously have multiple contracts with agents on the other side of the market and each agent has strict preferences over sets of contracts. Each contract specifies a firm, a worker, and some terms that may include a wage, benefits, etc (see Roth [1] and Hatfield and Milgrom [2] for models of matching with contracts). We focus on stable matchings, that is matchings in which each agent is willing to keep all of their contracts and there are no contracts that agents would like to sign, possibly by dropping some of their current contracts (stable matchings exist when contracts are substitutes Roth [1], Hatfield and Milgrom [2], Fleiner [3], Klaus and Walzl [4], Hatfield and Kominers [5]. Contracts are substitutes when a contract that is chosen from a larger set is also chosen from a smaller subset which includes that contract).

We introduce quantile stable mechanisms: a new class of matching mechanisms that generate stable matchings that can be seen as a compromise between two sides of the market, and we study their properties. Two of the quantile-stable mechanisms are well known and often used: the side-optimal (or, extremal) matching mechanisms that assign the best stable outcome for one side and the worst for the other. Extremal mechanisms have been implemented, for instance, in the National Resident Matching Program (NRMP) to match medical doctors to residency programs Roth [6] and in some school districts to match students to high schools Abdulkadiroğlu and Sönmez [7]. As far as we know, none of the non-extremal quantile stable mechanisms has been implemented.

Among the non-extremal quantile stable mechanisms, the median stable mechanism that produces the median stable matching is a focal matching mechanism a market designer may want to implement: it is attractive since it may be seen as a compromise solution that treats both sides of the market in a symmetric way. Indeed, experiments performed by Echenique and Yariv [8] support this view as they show that agents who match in a decentralized way tend to coordinate on a particular stable matching, the median stable matching, when it exists (Echenique and Yariv [8] show that the median stable matching is selected most frequently by the subjects and that the cardinal representation of ordinal preferences also impacts which stable matching gets selected. The experimental bargaining literature also reports that subjects choose the median split, e.g., Anbarci and Feltovich [9]. 
Even in settings where the core prediction is that the entire surplus goes to one side of the market, subjects have a strong propensity towards equal splits, Yan et al. [10]. We would like to thank an anonymous referee for directing us to the latter two papers). The median stable mechanism is also as good as the extremal mechanisms from the perspective of manipulability by both sides of the market; indeed, our Proposition 3 shows that no quantile stable mechanism is more manipulable than another when we account for agents on both sides of the market.

The quantile stable mechanisms are defined as follows. For any $q \in(0,1]$ the $q$-quantile stable mechanism maps agents' preference profiles into the $\lceil\mathrm{kq}\rceil$ best stable matching for either firms or workers, where $k$ is the number of stable matchings ( $\lceil x\rceil$ is the smallest integer that is weakly larger than $x$. An analogous theory is true for $q$-quantile stable mechanisms defined in terms of the largest integer that is weakly smaller than $\mathrm{kq}$ ). For instance, an extremal mechanism may always select the worker-optimal stable matching, and, given $k$ stable matchings, the median stable mechanism always selects the matching that is the $\left\lceil\frac{k}{2}\right\rceil$-best for workers (and hence $\left\lfloor\frac{k}{2}\right\rfloor$-best for firms).

For the quantile stable mechanisms to be well-defined we need to know that $\lceil k q\rceil$-best stable matchings exist. We call such matchings, quantile stable matchings. Teo and Sethuraman [11], Fleiner [12], Schwarz and Yenmez [13] proved the existence of quantile stable matchings in one-to-one matching, and Klaus and Klijn [14] and Sethuraman et al. [15] proved it for responsive preferences in the college admission model; in this model, preferences are responsive if each agent's preferences over sets of partners on the other side reflects an underlying ranking of individual partners (in addition to the finite case, Schwarz and Yenmez [13] study a continuum of potential wages. Cf. also Klaus and Klijn [16] who study the existence of quantile stable matchings in the roommates problem). In the general matching-with-contracts model, Chen et al. [17] showed that the existence of quantile stable matchings is guaranteed by two properties of agents' preferences: strong substitutes and the law of aggregate demand (between 2014 and 2016, Chen et al. [17] used to be Section 3.1 of the working-paper draft of the present paper, cf., e.g., Chen et al. [18]. Chen et al. [18] and Chen et al. [19] subsumed Chen et al. [20], which is obsolete now. The resulting three papers—-the present paper, Chen et al. [17], and Chen et al. [19]—cross-reference and complement each other. Each has a distinct focus and a distinct set of results). Contracts are strong substitutes if a contract chosen from a set of contracts is also chosen from any worse set of contracts including that contract, cf. Echenique and Oviedo [21]. Contracts satisfy the law of aggregate demand if the number of contracts chosen from a larger set is weakly greater than the number of contracts chosen from a smaller set, cf. Hatfield and Milgrom [2].

We complement Chen et al. [17] by showing that both strong substitutes and the law of aggregate demand are needed in their sufficiency condition, and by observing that strong substitutes and the law of aggregate demand are satisfied in natural models of job assignment, including the large firms model studied in Eeckhout and Kircher [22]. We also contribute to the existence literature discussed above by showing that the responsiveness of preferences is a sufficient condition for the existence of quantile stable matchings not only in college admission, but also in the matching with contracts model we study (see Proposition 1) (except in one-to-one settings, responsive preferences do not necessarily satisfy strong substitutes (cf. Example 6.8 in Echenique and Oviedo [21]), and thus neither our general Proposition 1 nor the the college admission results of Klaus and Klijn [14] and Sethuraman et al. [15] follow directly from Chen et al. [17]).

Our Proposition 2 shows that all quantile stable mechanisms are distinct in sufficiently large markets. Finally, in Proposition 3 we tackle the manipulability of the quantile stable mechanisms. Following Day and Milgrom [23], Pathak and Sönmez [24], and Chen et al. [19], we say that a mechanism $\psi$ is as manipulable as mechanism $\phi$ for an agent if whenever the agent can gain from misreporting in $\phi$ and achieve a certain outcome, she can also gain and achieve this outcome by manipulating $\psi$. A mechanism $\psi$ is more manipulable than mechanism $\phi$ for an agent if it is as manipulable and in addition there 
exists an instance of the market in which she can manipulate $\psi$ but not $\phi$. Leveraging the results of Chen et al. [19], we show that quantile stable mechanisms can be naturally ranked for each side of the market in terms of how manipulable they are. In particular, as we choose a higher quantile for one side of the market, we make the mechanism less manipulable for that side, but more manipulable for the other side of the market (note that the mechanism is then choosing a lower quantile for the other side of the market). Thus, no quantile stable matching is better than another in terms of manipulability when agents on both sides of the market are strategic.

To the best of our knowledge ours is the first paper to study quantile stable mechanisms other than deferred acceptance, and, in particular, the first to study their incentive properties.

\section{Model}

We study the same setting as our companion papers Chen et al. [17] and Chen et al. [19]. The set of agents is $A$ and it is partitioned into the set of firms $F$ and the set of workers $W$. Each contract $x$ specifies a bilateral relationship between a firm and a worker. We denote the firm associated with contract $x$ by $x_{F} \in F$ and the worker associated with this contract by $x_{W} \in W$. The set of all contracts, denoted $X$, is finite. For a set of contracts $X^{\prime} \subseteq X$, $X_{a}^{\prime} \equiv\left\{x \mid x \in X^{\prime}, a \in\left\{x_{F}, x_{W}\right\}\right\}$ denotes the set of contracts that agent $a$ is associated with. A set of contracts $X^{\prime}$ is feasible if for every firm-worker pair $(f, w) \in F \times W,\left|X_{f}^{\prime} \cap X_{w}^{\prime}\right| \leq 1$, i.e., each firm-worker pair can sign at most one joint contract. A matching is a feasible set of contracts.

Each agent $a$ is endowed with a strict preference relation $\succ_{a}$ over sets of contracts that involve agent $a$, i.e., over $2^{X_{a}} \equiv\left\{X^{\prime} \mid X^{\prime} \subseteq X_{a}\right\}$. Agent $a^{\prime}$ s weak preference relation is denoted by $\succeq_{a}$; for all $Y, Y^{\prime} \subseteq X_{a}, Y \succeq_{a} Y^{\prime}$ if and only if $Y \succ_{a} Y^{\prime}$ or $Y=Y^{\prime}$. Given $\succeq_{a}$, let $C_{a}\left(X^{\prime} \mid \succeq_{a}\right)$ denote agent $a^{\prime}$ s most preferred subset of contracts involving agent $a$ from $X^{\prime}$. More formally, $C_{a}\left(X^{\prime} \mid \succeq_{a}\right) \subseteq X_{a}^{\prime}$ and for all $Y_{a} \subseteq X_{a}^{\prime}, C_{a}\left(X^{\prime} \mid \succeq_{a}\right) \succeq_{a} Y_{a}$. In notation throughout the paper, we suppress the dependence of $C_{a}$ on $\succeq_{a}$ when this does not lead to ambiguity. $C_{a}\left(X^{\prime}\right)$ denotes the set of contracts chosen from $X^{\prime} \subseteq X$. For any set of contracts $X^{\prime}$, we denote by $C_{W}\left(X^{\prime}\right) \equiv \cup_{w} C_{w}\left(X^{\prime}\right)$ and $C_{F}\left(X^{\prime}\right) \equiv \cup_{f} C_{f}\left(X^{\prime}\right)$ the sets of contracts chosen by the sets of workers and firms, respectively. A contract $x$ is called acceptable to agent $a$ if there exists a set of contracts $X_{a} \ni x$ such that $X_{a} \succ_{a} \varnothing$; otherwise contract $x$ is unacceptable to $a$.

Our solution concept is stability (see, e.g., Hatfield and Kominers [5]).

Definition 1. Given a preference profile $\succ$, a matching $Y$ is stable if

1. for all $a, C_{a}(Y)=Y_{a}$ (individual rationality) and

2. there does not exist a nonempty set of contracts $Z \nsubseteq \nsubseteq Y$ such that for all $a, Z_{a} \subseteq C_{a}(Y \cup Z)$ (no blocking).

Individual rationality requires that each agent is better off by holding all of the contracts assigned rather than rejecting some of them. No blocking requires that there is no subset of contracts $Z \nsubseteq Y$ such that every agent $a$ would choose $Z_{a}$ from the union of $Y$ and $Z$.

The following assumptions on agents' preferences play a role in our analysis.

Definition 2. Contracts are substitutes in preferences of agent $a$ if for any contract $x \in X$ and sets of contracts $Y, Y^{\prime} \subseteq X$ such that $Y \subseteq Y^{\prime}$,

$$
x \in C_{a}\left(\{x\} \cup Y^{\prime}\right) \Rightarrow x \in C_{a}(\{x\} \cup Y) .
$$

Contracts are substitutes when a contract that is chosen from a set is also chosen from any subset that includes the contract. Substitutability guarantees the existence of a stable matching in many-to-many matching models, cf. Fleiner [3], and it is one of the main restrictions on preferences in matching theory (this condition was introduced by 
Kelso and Crawford [25], and it corresponds to the independence-of-irrelevant-alternatives assumption (or Sen's property alpha) in individual choice theory. When choice rules are taken as primitives, substitutability needs to be combined with an axiom called the irrelevance of rejected contracts in order to ensure the existence of stable matchings, $\mathrm{cf}$. Aygün and Sönmez [26]. This latter axiom is embedded in our setup because we construct choice rules from agents' preferences over sets of contracts).

Definition 3. Contracts are strong substitutes in preferences of agent a if for any sets of contracts $Y, Y^{\prime} \subseteq X$ such that $C_{a}\left(Y^{\prime}\right) \succeq_{a} C_{a}(Y)$,

$$
x \in C_{a}\left(\{x\} \cup Y^{\prime}\right) \Rightarrow x \in C_{a}(\{x\} \cup Y) .
$$

Strong substitutability implies substitutability. Roughly, it states that if a contract is added to the two sets and chosen from the better set, then it must also be chosen from the worse set. This concept was first studied by Echenique and Oviedo [21] in their analysis of matching markets without contracts (as in Echenique and Oviedo [21], our results remain valid if we relax the strong substitutes condition to require only that: for any sets of contracts $Y, Y^{\prime} \subseteq X$ such that $C_{a}\left(Y^{\prime}\right)=Y_{a}^{\prime}, C_{a}(Y)=Y_{a}$, and $C_{a}\left(Y^{\prime}\right) \succeq_{a} C_{a}(Y)$, if $x \in C_{a}\left(Y^{\prime} \cup\{x\}\right)$ then $\left.x \in C_{a}(Y \cup\{x\})\right)$.

Definition 4. Contracts satisfy the law of aggregate demand in preferences of agent a if for all $Y, Y^{\prime} \subseteq X$ such that $Y \subseteq Y^{\prime}$

$$
\left|C_{a}(Y)\right| \leq\left|C_{a}\left(Y^{\prime}\right)\right| .
$$

The law of aggregate demand requires that the number of contracts chosen from a set is bigger than the number of contracts chosen from a subset of this set. The law of aggregate demand was introduced in Alkan [27], Alkan and Gale [28], and Fleiner [3] and its implications were thoroughly analyzed by Hatfield and Milgrom [2] (see also Kojima [29]).

Although the class of environments in which contracts are strong substitutes and satisfy the law of aggregate demand is limited, it encompasses some interesting examples. For instance, Hatfield and Milgrom [2] show that if contracts specify monetary payments and agents' preferences are quasi-linear, then the law of aggregate demand is satisfied. Hatfield and Milgrom [2] also provide the endowed assignment model in which contracts are substitutes in addition to satisfying the law of aggregate demand. The example below shows that in a natural special case of the endowed assignment model contracts are not only substitutes but also strong substitutes.

Example 1. Consider a many-to-one matching market between firms and workers. Each firm has a technology and a set of jobs to fill. A worker can fill any of a firm's jobs but no firm can use one worker for two jobs, nor assign two or more workers to the same job. A contract specifies the firm, the job, the worker, and the wage transfer. Each worker-job pair generates an output; this output does not depend on what other workers the firm employs or how it assigns them to jobs. The firm's payoff is the sum of outputs from its different jobs, net of wage transfers. If all available contracts for a particular job at a firm yield negative net output, then the firm leaves that job unfilled. Workers choose among contracts based on the wages offered; if no non-negative wage contract is available, then the worker accepts none.

To date, we have described the endowment assignment model of Hatfield and Milgrom [2]. In their model, contracts are substitutes and satisfy the law of aggregate demand, but the strong substitutes condition may fail. To guarantee that this last condition is satisfied, we assume that each worker is characterized by a one-dimensional ability parameter. If the worker's output in a job depends only on the job and the worker's ability (but not on their identity otherwise), and if output is increasing in worker's ability, then contracts are strong substitutes (Eeckhout and Kircher [22] study a continuous version of this example. In particular, taking the limit of our model in the case of quasi-linear preferences with monetary increments, like in Schwarz and Yenmez [13], one can use 
our results to show that the set of competitive equilibria in Eeckhout and Kircher [22] has a quantile structure. We can also generalize this example to a many-to-many matching market. For further analysis of the endowment assignment model, see Delacretaz et al. [30]).

\section{Quantile Stable Matchings}

As a preparation to study the quantile stable mechanisms, let us briefly review and further develop the theory of the quantile stable matchings from Chen et al. [17]. Suppose that $\left\{X^{1}, \ldots, X^{k}\right\}$ is the set of stable matchings. For each agent $a$, consider the set of contracts that agent $a$ signs in these matchings: $\left\{X_{a}^{1}, \ldots, X_{a}^{k}\right\}$. Reorder these sets of contracts so that $X_{a}^{(1)} \succeq_{a} \ldots \succeq_{a} X_{a}^{(k)}$. Let $X_{W}^{i} \equiv \cup_{w \in W} X_{w}^{(i)}$ and $X_{F}^{i} \equiv \cup_{f \in F} X_{f}^{(i)}$ for $1 \leq i \leq k$. That is, $X_{W}^{i}$ assigns each worker the $i$-th best outcome among all stable matching outcomes, and $X_{F}^{i}$ assigns each firm the $i$-th best outcome among all stable matching outcomes.

Chen et al. [17] showed that $X_{W}^{i}$ are well-defined stable matchings provided contracts are substitutes, satisfy the law of aggregate demand for all agents, and are strong substitutes for workers (as strong substitutes are satisfied when workers have unit demand and satisfy the standard substitute condition, the existence then condition then takes a simpler form). Furthermore, for any firm $f, X_{W}^{i} \succeq_{f} X_{W}^{j}$ if $i \geq j$. An analogous results holds true for $X_{F}^{i}$. Their existence result allows us to call $X_{W}^{i}$ the $i$-th quantile stable matching for workers. In particular, $X_{W}^{1}$ is the worker-optimal stable matching and $X_{W}^{k}$ is the workerpessimal stable matching. In the analogous case, when contracts are strong substitutes for firms, we define the quantile, firm-optimal, and firm-pessimal stable matchings for firms. Furthermore, the quantile stable matchings for firms and workers are exactly the same with the polarization of interests property, $X_{W}^{i}=X_{F}^{k+1-i}$, when contracts are strong substitutes for both workers and firms. Thus, the worker-optimal stable matching is the firm-pessimal stable matching, the (2)-nd quantile stable matching for workers is the $(k-1)$-th quantile stable matching for firms, etc. In particular, when $k$ is odd, there exists a stable matching that assigns all agents their median stable matching outcomes since $X_{F}^{(k+1) / 2}=X_{W}^{(k+1) / 2}$.

We complement Chen et al. [17] by showing that both their conditions are needed in their result: the quantile stable matchings other than the worker-optimal and firm-optimal stable matchings need not exist when either the strong substitute condition or the law of aggregate demand fails (the worker-optimal and firm-optimal stable matchings are exceptions. The lattice structure established in Alkan [27] and Fleiner [3] implies that the side-optimal stable matchings exist whenever contracts are merely substitutes and satisfy the law of aggregate demand). We establish the necessity of these two conditions via two examples presented in the Appendix A.

We also improve upon Klaus and Klijn [14] and Sethuraman et al. [15] by showing that responsiveness of preferences is sufficient for the existence of quantile stable matchings beyond the college admission model they study.

Definition 5. An agent a has responsive preferences over contracts if there exist a quota $q_{a}$ and a strict ordering $\gg_{a}$ on contracts $X_{a}$ and the empty contract $\varnothing$ such that (1) agent a prefers the empty set of contracts to any set of contracts $|Y|>q_{a}$, and (2) agent a prefers a set of contracts $Y \equiv\left\{y_{1}, \ldots, y_{|Y|}\right\} \subseteq X_{a}$ (indexed so that $\left.y_{1} \gg_{a} \ldots,>_{a} y_{|Y|}\right)$ to another set of contracts $Z \equiv\left\{z_{1}, \ldots, z_{|Z|}\right\} \subseteq X_{a}$ (indexed so that $\left.z_{1} \gg_{a} \ldots, \rightarrow_{a} z_{|Y|}\right)$ such that $Y \neq Z$ whenever $|Y| \leq q_{a}$ and either

- $|Y| \geq|Z|, y_{i} \gg_{a} z_{i}$ or $y_{i}=z_{i}$ for every $1 \leq i \leq|Z|$, and $y_{i} \gg_{a} \varnothing$ for every $|Z|+1 \leq i \leq|Y|$, or

- $|Y| \leq|Z|$ and $y_{i} \gg_{a} z_{i}$ or $y_{i}=z_{i}$ for every $1 \leq i \leq|Y|$ and $\varnothing \gg_{a} z_{i}$ for every $|Y|+1 \leq i \leq$ $|Z|$.

If agent $a$ has responsive preferences then there is an order over individual contracts and from each set of contracts agent $a$ chooses the best contracts without exceeding its quota. Note that a contract $x$ is acceptable for agent $a$ if $x>_{a} \varnothing$. 
Proposition 1. If all agents' preferences are responsive, then for all $i$ between 1 and the number of stable matchings $k$, the contract sets $X_{W}^{i}$ and $X_{F}^{i}$ are stable matchings; moreover, $X_{W}^{i}=X_{F}^{k+1-i}$.

This result does not directly follow from Chen et al. [17] because strong substitutes may fail for responsive preferences; cf. Example 6.8 in Echenique and Oviedo [21]. Still we are able to prove the proposition by reducing it to the results of Chen et al. [17]. The structure of the reduction has the same steps as the argument in Roth and Sotomayor [31] in which they prove that the classical lattice structure of the marriage problem carries over to the college admissions problem when colleges have responsive preferences. We first create an auxiliary one-to-one matching problem with contracts. In this auxiliary problem, the set of agents consists of $q_{a}$ numbered copies of each agent $a$ from the original problem; for each pair of replicas of agents $f$ and $w$ from two sides of the market, the set of contracts they can sign is isomorphic to the set of contracts $f$ and $w$ can sign in the original problem. We construct agents' preferences in the auxiliary problem as follows. Take an agent $\hat{a}$, who is a replica of agent $a$ in the original problem. Take any two contracts $\hat{x}$ and $\hat{y}$ created from contracts $x, y \in X_{a}$. If $x \succ_{a} y$ in the original problem, then $\hat{x} \succ_{\hat{a}} \hat{y}$ in the auxiliary problem. If $x$ is identical to $y$ in the original problem, then the contracts $\hat{x}$ and $\hat{y}$ are with two different numbered replicas of the same original-problem-agent, and agent $\hat{a}$ ranks the two contracts according to the numbers of the replicas. For the auxiliary one-to-one matching problem with contracts, Theorem 2 of Chen et al. [17] implies that there is a lattice structure on stable matchings. Following the same steps as Roth and Sotomayor [31] we then conclude that this lattice induces a lattice on stable matchings in the original problem.

\section{Quantile Stable Mechanisms}

Finally, let us define the quantile stable mechanisms and examine their incentive properties. Fix one side of the matching market, say firms. For each $q \in(0,1]$, the q-quantile stable mechanism $\varphi^{q}$ is the mapping from agents' preference profiles to matchings such that for every preference profile $\succ$, the mechanism $\varphi^{q}(\succ)$ selects the $\lceil k q\rceil$-th quantile stable matching for firms where $k$ is the number of stable matchings under $\succ$. Here, $\lceil x\rceil$ denotes the lowest integer equal to or larger than $x$ (all our results remain valid for mechanisms that always select the $\lfloor k q\rfloor$-th quantile stable matching, where $\lfloor x\rfloor$ is the highest integer smaller than or equal to $x$ ).

In light of the discussion above, the quantile stable mechanisms are well defined when we restrict attention to the domain of preferences that satisfy strong substitutes and the law of aggregate demand, and Proposition 1 shows that they are also well defined on the domain of responsive preferences. In what follows we assume that the quantile stable mechanisms we study are defined on one of these two preference domains.

We first show that all quantile stable mechanisms are different:

Proposition 2. For any $q, q^{\prime} \in(0,1]$ such that $q \neq q^{\prime}$, there exists a matching market such that $\varphi^{q}$ is different than $\varphi^{q^{\prime}}$.

To prove this proposition, it is sufficient to consider the case $q>q^{\prime}$ as the other case is symmetric. Let $k$ be such that $k\left(q-q^{\prime}\right)>1$. Consider the following market: All agents have unit demand and every firm-worker pair uniquely defines a contract. Let firm $f_{i}$ rank workers as follows $w_{i} \succ_{f_{i}} w_{i+1} \succ_{f_{i}} \ldots \succ_{f_{i}} w_{i+k-1}$ (subscripts are added modulo $k$ ) and let worker $w_{i+k-1}$ rank firms as follows $f_{i} \succ_{w_{i+k-1}} f_{i+1} \succ_{w_{i+k-1}} \ldots \succ_{w_{i+k-1}} f_{i+k-1}$. Under this preference profile there are $k$ distinct stable matchings and all quantile stable matchings are different. In this market, the $q$-quantile stable mechanism is different from the $q^{\prime}$-quantile stable mechanism since $k\left(q-q^{\prime}\right)>1$.

Finally, we study the manipulability properties of quantile stable matchings using Definition 5 from Chen et al. [19]. Fix a mechanism $\phi$. We need the following notation for the set of improvements,

$$
M(a, \phi, \succ)=\left\{\phi\left(\succ_{a}^{\prime} \succ_{-a}\right) \mid \succ_{a}^{\prime} \in P_{a} \text { and } \phi\left(\succ_{a}^{\prime}, \succ_{-a}\right) \succ_{a} \phi(\succ)\right\},
$$


which is comprised of improvements that agent $a$ can obtain by misreporting to mechanism $\phi$ when the preference profile is $\succ$. Mechanism $\psi$ is as manipulable as mechanism $\phi$ for agent $a$ if

$$
M(a, \psi, \succ) \supseteq M(a, \phi, \succ)
$$

for every preference profile $\succ \in P$. Mechanism $\psi$ is more manipulable than mechanism $\phi$ for agent $a$ if $\psi$ is as manipulable as $\phi$ for agent $a$ and there exists a preference profile $\succ \in P$ such that

$$
M(a, \psi, \succ) \supsetneq M(a, \phi, \succ) .
$$

As Chen et al. [19] discuss, this definition of manipulability comparison is equivalent to manipulability comparisons studied by Day and Milgrom [23] and Pathak and Sönmez [24]. The latter paper also studies less demanding manipulability concepts, and a fortiori our Proposition 3 remains valid for these less demanding concepts.

Proposition 3. Let $q, q^{\prime} \in(0,1]$ be such that $q>q^{\prime}$. Then either

- $\varphi^{q}=\varphi^{q^{\prime}}$,or

- $\quad \varphi^{q}$ is more manipulable than $\varphi^{q^{\prime}}$ for all firms and $\varphi^{q^{\prime}}$ is more manipulable than $\varphi^{q}$ for all workers.

This proposition follows from Chen et al.'s (2016) polarity result discussed above (for the preference domain satisfying strong substitutes and the law of aggregate demand) and from our Proposition 1 (for the domain of responsive preferences) when combined with the equivalence of manipulability and side-welfare established in Chen et al. [19]. To apply the latter equivalence we need to observe that each of the above two preference domains is closed in the sense of Chen et al. [19]: A preference profile domain $P$ is closed if for all $\succ \in P$ and for all matchings $Y$ that are stable with respect to $\succ$, if the preference relation $\succ_{a}^{\prime}$ ranks sets of contracts in the same way as $\succ_{a}$ except that only contracts in $Y_{a}$ are acceptable to agent $a$, then $\left(\succ_{a}^{\prime}, \succ_{-a}\right) \in P$.

\section{Conclusions}

We defined the class of quantile-stable mechanisms and analyzed their properties. These mechanisms are further studied by Fernandez [32], as part of their analysis of the absence and presence of regret in matching mechanisms.

Author Contributions: The authors contributed equally to this work. All authors have read and agreed to the published version of the manuscript.

Funding: Pycia gratefully acknowledges the support of UCLA, where he was on faculty while working on this paper. Yenmez gratefully acknowledges financial support from National Science Foundation grant SES-1326584 and the support of the Tepper School of Business, Carnegie Mellon University, where he was on faculty while working on this paper.

Institutional Review Board Statement: Not applicable.

Informed Consent Statement: Not applicable.

Acknowledgments: For useful comments, we would like to thank Federico Echenique, Parag Pathak, and Peng Wang.

Conflicts of Interest: The authors declare no conflict of interest.

\section{Appendix A. Necessity Examples}

In the following example, we show that strong substitutes cannot be replaced with substitutes in the existence results of Chen et al. [17].

Example A1. There are two firms $f_{1}, f_{2}$; and four workers $w_{1}, w_{2}, w_{3}$, and $w_{4}$. Workers have unit demand. There is only one contract that each firm-worker pair can sign. To ease notation, 
each contract is denoted by the pair of agents associated with this contract. Let $x_{1}=\left\{f_{1}, w_{1}\right\}$, $x_{2}=\left\{f_{1}, w_{2}\right\}, x_{3}=\left\{f_{1}, w_{3}\right\}, x_{4}=\left\{f_{1}, w_{4}\right\}, x_{5}=\left\{f_{2}, w_{1}\right\}, x_{6}=\left\{f_{2}, w_{2}\right\}, x_{7}=\left\{f_{2}, w_{3}\right\}$, and $x_{8}=\left\{f_{2}, w_{4}\right\}$. Preferences are as follows: (A set of contracts $\left\{x_{i}, \ldots, x_{j}\right\}$ is denoted by $x_{i} \ldots x_{j}$ to ease notation. If a set of contracts is omitted from the preference list of an agent, the agent prefers the null contract to that set of contracts).

$$
\begin{aligned}
& \succeq_{f_{1}}: x_{1} x_{3}, x_{1} x_{4}, x_{2} x_{3}, x_{2} x_{4}, x_{1}, x_{3}, x_{2}, x_{4} ; \\
& \succeq_{f_{2}}: x_{6} x_{8}, x_{5} x_{8}, x_{6} x_{7}, x_{5} x_{7}, x_{6}, x_{8}, x_{5}, x_{7} ; \\
& \succeq_{w_{1}}: x_{5}, x_{1} ; \\
& \succeq_{w_{2}}: x_{2}, x_{6} ; \\
& \succeq_{w_{3}}: x_{7}, x_{3} ; \text { and } \\
& \succeq_{w_{4}}: x_{4}, x_{8} .
\end{aligned}
$$

Note that contracts are substitutes and satisfy the law of aggregate demand for all agents. Moreover, they are also strong substitutes for workers and $f_{2}$. However, contracts are not strong substitutes for $f_{1}$ because even though $x_{1} x_{4} \succeq_{f_{1}} x_{2} x_{3}$, and $x_{4} \in C_{f_{1}}\left(\left\{x_{1}, x_{4}\right\} \cup\left\{x_{4}\right\}\right)$ we have $x_{4} \notin C_{f_{1}}\left(\left\{x_{2}, x_{3}\right\} \cup\left\{x_{4}\right\}\right)=\left\{x_{2}, x_{3}\right\}$.

There are four stable matchings: $\mu_{1} \equiv\left\{x_{1}, x_{3}, x_{6}, x_{8}\right\}, \mu_{2} \equiv\left\{x_{1}, x_{4}, x_{6}, x_{7}\right\}, \mu_{3} \equiv$ $\left\{x_{2}, x_{3}, x_{5}, x_{8}\right\}$, and $\mu_{4} \equiv\left\{x_{2}, x_{4}, x_{5}, x_{7}\right\}$. When we use the quantile construction above we get: $X_{F}^{1}=\left\{x_{1}, x_{3}, x_{6}, x_{8}\right\}=\mu_{1}, X_{F}^{2}=\left\{x_{1}, x_{4}, x_{5}, x_{8}\right\}, X_{F}^{3}=\left\{x_{2}, x_{3}, x_{6}, x_{7}\right\}, X_{F}^{4}=\left\{x_{2}, x_{4}, x_{5}, x_{7}\right\}$ $=\mu_{4} ;$ and $X_{W}^{1}=\left\{x_{2}, x_{4}, x_{5}, x_{7}\right\}=\mu_{1}, X_{W}^{2}=\left\{x_{2}, x_{4}, x_{5}, x_{7}\right\}=\mu_{1}, X_{W}^{3}=\left\{x_{1}, x_{3}, x_{6}, x_{8}\right\}=$ $\mu_{4}, X_{W}^{4}=\left\{x_{1}, x_{3}, x_{6}, x_{8}\right\}=\mu_{4}$. Here $X_{F}^{2}$ and $X_{F}^{3}$ are not even matchings, let alone stable.

As the following example shows, the law of aggregate demand is also necessary in the existence results of Chen et al. [17]. In the following example contracts are strong substitutes for all agents but the law of aggregate demand fails. There are four stable matchings including the firm-optimal and the worker-optimal stable matchings, but other quantile stable matchings do not exist (this example develops Example 5 of Alkan and Gale [28] who use it to show that the law of aggregate demand is necessary to get the lattice structure of stable matchings).

Example A2. There are five firms $f_{1}, f_{2}, f_{3}, f_{4}, f_{5}$; and five workers $w_{1}, w_{2}, w_{3}, w_{4}$, and $w_{5}$. Workers have unit demand. For each firm-worker pair, there is only one contract that they can sign. To ease notation, each contract is denoted by the pair of agents associated with this contract. Let $x_{1}=\left\{f_{1}, w_{1}\right\}, x_{2}=\left\{f_{1}, w_{3}\right\}, x_{3}=\left\{f_{1}, w_{5}\right\}, x_{4}=\left\{f_{2}, w_{2}\right\}, x_{5}=\left\{f_{2}, w_{4}\right\}, x_{6}=\left\{f_{2}, w_{5}\right\}$, $x_{7}=\left\{f_{3}, w_{3}\right\}, x_{8}=\left\{f_{3}, w_{1}\right\}, x_{9}=\left\{f_{4}, w_{4}\right\}, x_{10}=\left\{f_{4}, w_{2}\right\}$, and $x_{11}=\left\{f_{5}, w_{5}\right\}$. Preferences are as follows:

$$
\begin{aligned}
& \succeq_{f_{1}}: x_{1}, x_{2} x_{3}, x_{2}, x_{3} ; \\
& \succeq_{f_{2}}: x_{4}, x_{5} x_{6}, x_{5}, x_{6} ; \\
& \succeq_{f_{3}}: x_{7}, x_{8} ; \\
& \succeq_{f_{4}}: x_{9}, x_{10} ; \\
& \succeq_{f_{5}}: x_{11} ; \\
& \succeq_{w_{1}}: x_{8}, x_{1} ; \\
& \succeq_{w_{2}}: x_{10}, x_{4} ; \\
& \succeq_{w_{3}}: x_{2}, x_{7} \\
& \succeq_{w_{4}}: x_{5}, x_{9} ; \text { and } \\
& \succeq_{w_{5}}: x_{3}, x_{6}, x_{11} .
\end{aligned}
$$

Since workers $f_{3}, f_{4}$, and $f_{5}$ have unit demand, contracts are strong substitutes and satisfy the law of aggregate demand for these agents. However, even though contracts are strong substitutes 
for $f_{1}$ and $f_{2}$, they do not satisfy the law of aggregate demand for these two firms. This is straightforward. For example, for $f_{1}, C_{f_{1}}\left(\left\{x_{1}, x_{2}, x_{3}\right\}\right)=x_{1}$ and $C_{f_{1}}\left(\left\{x_{2}, x_{3}\right\}\right)=\left\{x_{2}, x_{3}\right\}$, which imply $\left|C_{f_{1}}\left(\left\{x_{1}, x_{2}, x_{3}\right\}\right)\right|<\left|C_{f_{1}}\left(\left\{x_{2}, x_{3}\right\}\right)\right|$.

There are four stable matchings: $\mu_{1} \equiv\left\{x_{1}, x_{4}, x_{7}, x_{9}, x_{11}\right\}, \mu_{2} \equiv\left\{x_{1}, x_{5}, x_{6}, x_{7}, x_{10}\right\}, \mu_{3} \equiv$ $\left\{x_{2}, x_{3}, x_{4}, x_{8}, x_{9}\right\}$, and $\mu_{4} \equiv\left\{x_{2}, x_{3}, x_{5}, x_{8}, x_{10}\right\}$. When we use the quantile construction above we get: $X_{F}^{1}=\left\{x_{1}, x_{4}, x_{7}, x_{9}, x_{11}\right\}=\mu_{1}, X_{F}^{2}=\left\{x_{1}, x_{4}, x_{7}, x_{9}\right\}, X_{F}^{3}=\left\{x_{2}, x_{3}, x_{5}, x_{6}, x_{8}, x_{10}\right\}, X_{F}^{4}=$ $\left\{x_{2}, x_{3}, x_{5}, x_{8}, x_{10}\right\}=\mu_{4}$; and $X_{W}^{1}=\left\{x_{2}, x_{3}, x_{5}, x_{8}, x_{10}\right\}=\mu_{4}, X_{W}^{2}=\left\{x_{2}, x_{3}, x_{5}, x_{8}, x_{10}\right\}=$ $\mu_{4}, X_{W}^{3}=\left\{x_{1}, x_{4}, x_{6}, x_{7}, x_{9}\right\}, X_{W}^{4}=\left\{x_{1}, x_{4}, x_{7}, x_{9}, x_{11}\right\}=\mu_{4}$. Here $X_{F}^{2}$ is not stable since $x_{11}$ is a blocking contract, $X_{F}^{3}$ is not stable because it is not individually rational for $w_{5}$ as $C_{w_{5}}\left(\left\{x_{3}, x_{6}\right\}\right)=x_{3}$, and $X_{W}^{3}$ is not stable because it is not individually rational for $f_{2}$ as $C_{f_{2}}\left(\left\{x_{4}, x_{6}\right\}\right)=x_{4}$.

\section{References}

1. Roth, A.E. Stability and Polarization of Interests in Job Matching. Econometrica 1984, 52, 47-57. [CrossRef]

2. Hatfield, J.; Milgrom, P. Matching with Contracts. Am. Econ. Rev. 2005, 95, 913-935. [CrossRef]

3. Fleiner, T. A Fixed-Point Approach to Stable Matchings and Some Applications. Math. Oper. Res. 2003, 28, 103-126. [CrossRef]

4. Klaus, B.; Walzl, M. Stable many-to-many matchings with contracts. J. Math. Econ. 2009, 45, 422-434. [CrossRef]

5. Hatfield, J.W.; Kominers, S.D. Contract Design and Stability in Many-to-Many Matching. Games Econ. Behav. 2017, 101, 78-97. [CrossRef]

6. Roth, A.E. The Evolution of the Labor Market for Medical Interns and Residents: A Case Study in Game Theory. J. Political Econ. 1984, 92, 991-1016. [CrossRef]

7. Abdulkadiroğlu, A.; Sönmez, T. School Choice: A Mechanism Design Approach. Am. Econ. Rev. 2003, 93, 729-747. [CrossRef]

8. Echenique, F.; Yariv, L. An Experimental Study of Decentralized Matching; Working Paper; Caltech: Pasadena, CA, USA, 2013.

9. Anbarci, N.; Feltovich, N. How Fully do People Exploit Their Bargaining Position? The Effects of Bargaining Institution and the 50-50 Norm. J. Econ. Behav. Organ. 2018, 145, 320-334. [CrossRef]

10. Yan, H.; Friedman, D.; Munro, D. An Experiment on a Core Controversy. Games Econ. Behav. 2016, 96, 132-144. [CrossRef]

11. Teo, C.P.; Sethuraman, J. The geometry of fractional stable matchings and its applications. Math. Oper. Res. 1998, $23,874-891$. [CrossRef]

12. Fleiner, T. Some Results on Stable Matchings and Fixed Points; Working Paper; Egrervary Research Group: Budapest, Hungary, 2002.

13. Schwarz, M.; Yenmez, M.B. Median Stable Matching for Markets with Wages. J. Econ. Theory 2011, 146, 619-637. [CrossRef]

14. Klaus, B.; Klijn, F. Median stable matching for college admissions. Int. J. Game Theory 2006, 34, 1. [CrossRef]

15. Sethuraman, J.; Teo, C.P.; Qian, L. Many-to-One Stable Matching: Geometry and Fairness. Math. Oper. Res. 2006, 31, 581-596. [CrossRef]

16. Klaus, B.; Klijn, F. Smith and Rawls share a room: Stability and medians. Soc. Choice Welf. 2010, 35, 647-667. [CrossRef]

17. Chen, P.; Egesdal, M.; Pycia, M.; Yenmez, M.B. Median Stable Matchings in Two-Sided Markets. Games Econ. Behav. 2016, 97, 64-69. [CrossRef]

18. Chen, P.; Egesdal, M.; Pycia, M.; Yenmez, M.B. Quantile Stable Mechanisms; SSRN Working Paper No. 2526505; SSRN: Rochester, NY, USA, 2015.

19. Chen, P.; Egesdal, M.; Pycia, M.; Yenmez, M.B. Manipulability of Stable Mechanisms. Am. Econ. J. Microecon. 2016, 8, 202-214. [CrossRef]

20. Chen, P.; Egesdal, M.; Pycia, M.; Yenmez, M.B. Ranking by Manipulability and Quantile Stable Mechanisms; Working Paper; UCLA: Los Angeles, CA, USA, 2012.

21. Echenique, F.; Oviedo, J. A Theory of Stability in Many-to-Many Matching Markets. Theor. Econ. 2006, 1, 233-273. [CrossRef]

22. Eeckhout, J.; Kircher, P. Assortative Matching with Large Firms: Span of Control over More Versus Better Workers. Econometrica 2018, 86, 85-132. [CrossRef]

23. Day, R.; Milgrom, P. Core-selecting Package Auctions. Int. J. Game Theory 2008, 36, 393-407. [CrossRef]

24. Pathak, P.; Sönmez, T. School Admissions Reform in Chicago and England: Comparing Mechanisms by Their Vulnerability to Manipulation. Am. Econ. Rev. 2013, 1031, 80-106. [CrossRef]

25. Kelso, A.S.; Crawford, V.P. Job Matching, Coalition Formation, and Gross Substitutes. Econometrica 1982, 50, 1483-1504. [CrossRef]

26. Aygün, O.; Sönmez, T. Matching with Contracts: Comment. Am. Econ. Rev. 2013, 103, 2050-2051. [CrossRef]

27. Alkan, A. A class of multipartner matching models with a strong lattice structure. Econ. Theory 2002, 19, 737-746. [CrossRef]

28. Alkan, A.; Gale, D. Stable schedule matching under revealed preference. J. Econ. Theory 2003, 112, 289-306. [CrossRef]

29. Kojima, F. The Law of Aggregate Demand and Welfare in Two-sided Matching. Econ. Lett. 2007, 99, 581-584. [CrossRef]

30. Delacretaz, D.; Loertscher, S.; Marx, L.M.; Wilkening, T. Two-sided allocation problems, decomposability, and the impossibility of efficient trade. J. Econ. Theory 2019, 179, 416-454. [CrossRef]

31. Roth, A.E.; Sotomayor, M.A.O. Two-Sided Matching: A Study in Game-Theoretic Modeling and Analysis; Econometric Society Monographs: Cambridge, UK, 1990.

32. Fernandez, M.A. Deferred Acceptance and Regret-Free Truth-Telling; Working Paper; John Hopkins University: Baltimore, MD, USA, 2020. 